\title{
Publisher's Note: Bulk-boundary correspondence in (3+1)-dimensional topological phases [Phys. Rev. B 94, 045113 (2016)]
}

Xiao Chen, Apoorv Tiwari, and Shinsei Ryu

(Received 2 August 2016; published 9 August 2016)

DOI: 10.1103/PhysRevB.94.079903

This paper was published online on 13 July 2016 with an incorrect reference. Reference [15] should read as "C.-T. Hsieh, G. Y. Cho, and S. Ryu, Phys. Rev. B 93, 075135 (2016)." The reference has been corrected as of 2 August 2016. The reference is correct in the printed version of the journal. 